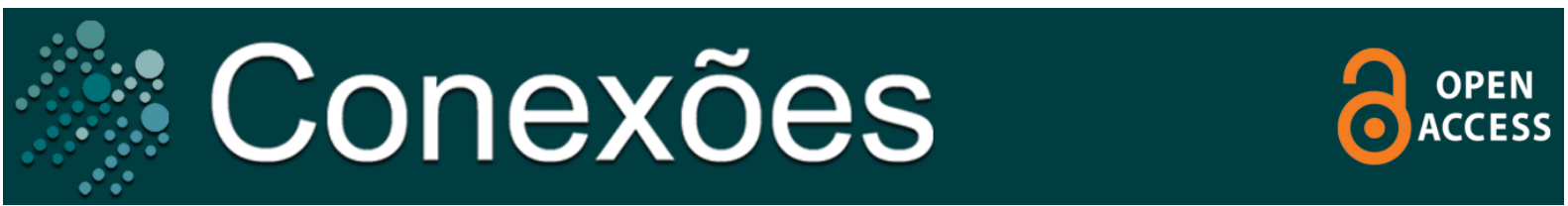

Artigo Original

\title{
Motivação em praticantes de exercício resistido
}

\author{
Motivation in resistance exercise practitioners \\ Motivación em practicantes de ejercicios de resistance \\ Elisabeth Cristina Canola ${ }^{1}$ \\ João Guilherme Cren Chiminazzo ${ }^{1}$
}

\begin{abstract}
RESUMO
Introdução: A importância da prática de exercícios físicos pela população tem se tornado objeto de estudo de diversas áreas, tendo se comprovado os benefícios oriundos da realização constante dos exercícios físicos. Para incorporar essa prática na rotina, se faz importante saber a motivação das pessoas pela busca de um local para a prática de exercícios físicos. Objetivo: avaliar os níveis motivacionais de praticantes de exercícios resistidos. Método: 94 adultos, praticantes de exercícios resistidos, responderam a dois questionários, sendo um sóciodemográfico e o Behavioral Regulation in Exercise Questionnaire - Version3 (BREQ-3). Resultados: 54,3\% dos participantes buscavam saúde ao praticar o exercício resistido, e em relação aos domínios do BREQ-3, a média para a motivação intrínseca foi a mais alta. Conclusão: os participantes do estudo buscam por saúde e, de uma forma geral, eles se encontram motivados intrinsecamente na prática de exercícios resistidos.
\end{abstract}

Palavras-chave: Exercício físico. Academias de Ginástica. Autonomia Pessoal.

${ }^{1}$ Centro Universitário de Jaguariúna, Jaguariúna - SP, Brasil.

Correspondência:

Elisabeth Cristina Canola. Rua Ítalo Avancini, 284, Bairro Bráz Cavenaghi, Itapira - SP, CEP 13976493. Email: elisabeth.canola@hotmail.com 


\begin{abstract}
Introduction: The importance of the practice of physical exercise by the population has become the object of study in several areas, with benefits arising from the constant performance of physical exercises. To incorporate this practice into the routine, it is important to know people's motivation for finding a place to practice physical exercises. Objective: assess the motivational levels of resistance exercise practitioners. Method: 94 adults, practitioners of resistance exercise, answered two questionnaires, one sociodemographic and the Behavioral Regulation in Exercise Questionnaire - Version3 (BREQ-3). Results: $54,3 \%$ of the participants sough health when practicing resistance exercise, and in relation to the BREQ-3 domains, the mean for intrinsic motivation was the highest. Conclusion: participants seek health and, in general, they find themselves intrinsically motivated in the practice of resistance exercises.
\end{abstract}

Keywords: Exercise. Fitness Centers. Personal Autonomy.

\title{
RESUMEN
}

Introducción: La importância de la práctica de ejercicios físicos por pare de la población se há convertido em objeto de estúdio em diversas áreas, siendo los benefícios derivados de la realización constante de ejercicios físicos. Para incorporar esta práctica a la rutina, es importante conocer la motivación de las personas para encontrar un lugar donde practicar ejercicios físicos. Objetivo: evaluar los niveles de motivación de los practiacntes de ejercicios de resistance. Método: 94 adultos, practicantes de ejercicio resistance, respondieron dos cuestionarios, uno sociodemográfico y el Cuestionario de Regulación Conductual en el Ejercicio - Versión 3 (BREQ-3). Resultados 54,3\% de los participantes buscaba la salud al practicar ejercicio de resistência, y em relación a los domínios BREQ3 , la media de motivación intrínseca fu ela más alta. Conclusion: los participantes de estúdio buscan la salud y, em general, se encuentran intrinsecamente motivados em la práctica de ejercicios de resistência.

Palabras Clave: Ejercicio fisico. Centros de Acondicionamiento. Autonomía Personal. 


\section{INTRODUÇÃO}

Diversas áreas têm se dedicado a estudar os benefícios à saúde decorrentes da prática regular e contínua do exercício físico (DECI, RYAN, 2014; RUFINI, BZUNECK, OLIVEIRA, 2012; RYAN, DECI, 2017), o que afirma a importância de as pessoas incorporarem hábitos saudáveis na sua rotina diária. Estudos têm comprovado que a realização constante de exercícios pode trazer benefícios ao praticante (MATSUDO; MATSUDO; NETO, 2000), que vão desde aspectos fisiológicos até benefícios psicológicos e sociais (BAVOSO et al., 2017, NAHAS, 2003, SILVA et al., 2010).

Para desfrutar destes benefícios é importante que haja um engajamento da prática de exercícios, desta forma, esta atividade deve ser prazerosa àqueles que a praticam (SAMULSKI; COSTA; AMPARO, 2009). Neste sentido, Williams et al. (2008) comprovaram associações positivas entre respostas afetivas referentes ao exercício e adesão às práticas, sendo que respostas afetivas são determinantes para a prática de exercícios.

Além do desafio de começar um programa de exercícios, conhecido também como adesão, outro ponto importante é o de manter-se no programa, conhecido como aderência (SABA, 2001; SANTOS; KNIJNIK, 2006). Marcus (2000) e Saba (2001) apontam que $50 \%$ das pessoas que começam um programa de prática de exercícios físicos desistem nos primeiros seis meses. Em academias de ginástica, esse dado é bastante nítido, visto que é notória a grande rotatividade de alunos nesses espaços (SANTOS; KNIJNIK, 2006). Com isso, é interessante investigar quais os motivos que levam os adultos a esses locais em busca da prática de exercícios sistematizados e qual a razão que os fazem permanecer exercitando-se de maneira contínua (CARNEIRO; GOMES, 2016).

A motivação é uma das habilidades psicológicas mais estudadas atualmente (FERREIRA, DE SOUZA, 2019) e pode ser entendida como um processo psicológico que auxilia a compreensão das diferentes escolhas de uma pessoa, sendo um dos fatores que determinam o comportamento da mesma (SCHULTZ; SCHULTZ, 2002). Para Deci e Ryan (1985) autores da Teoria da Autodeterminação (TAD) a motivação pode ser compreendida de forma global, considerando um continuum da forma mais autodeterminada para a menos autodeterminada, em motivação intrínseca, motivação extrínseca e amotivação (FERNANDES; RAPOSO, 2005), e os diferentes níveis de auto-regulação: externa, introjetada, identificada e integrada (RYAN; DECI, 2000).

Na TAD a motivação é intrínseca quando o ingresso na atividade ocorre por vontade própria, por prazer em realizar determinada prática e o anseio em se aprofundar na mesma (RYAN; DECI, 2000). Já a motivação externa, segundo os autores, ocorre quando o lócus de controle para a ação é externo à pessoa, sendo que ocorre através de quatro processos regulatórios: 1) regulação externa: tal 
comportamento é realizado para suprir uma demanda externa ou para receber algum tipo de recompensa (FERNANDES; RAPOSO, 2005); 2) regulação introjetada: trata-se de recompensas e punições internas, havendo sentimento de obrigação, ansiedade, ou orgulho (RYAN; DECI, 2000); 3) regulação identificada: comportamento regulado mais internamente, onde o indivíduo o considera importante e aprecia os resultados e os benefícios da participação em tal atividade; 4) regulação integrada: considerada extrínseca porque visa algum tipo de resultado além do prazer da prática (RYAN; DECI, 2000).

Estudos prévios identificaram que um dos principais motivos para a prática de exercícios resistidos está na busca pela saúde (SILVA et al., 2021; SANTOS et al., 2018). Além disso, a motivação intrínseca e a regulação identificada são os preditores motivacionais principais nas fases de aderência e adesão para atividades de exercícios resistidos (KOPP et al., 2020; MOUTÃo et al., 2012).

Nota-se um aumento do número de pessoas que procuram praticar exercícios físicos em academias de ginástica com a busca de uma melhor qualidade de vida e interação social (SILVA; PEREIRA; VENÂNCIO, 2016). Assim, reconhecendo o nível motivacional dessas pessoas, o profissional poderá atender de forma diferenciada tornando a motivação cada vez mais autônoma. Desta forma, o objetivo deste estudo foi analisar os níveis motivacionais de praticantes de exercício resistido.

\section{MÉTODO}

Participaram do estudo 94 adultos com idade entre 18 e 60 anos, sendo 53 homens (32,0 $\pm 9,6$ anos) e 41 mulheres $(35,12 \pm 10,85$ anos) que praticavam exercícios resistidos nas academias de ginástica escolhidas por conveniência e acessibilidade para participarem da pesquisa.

Nesse trabalho foi adotado o termo exercício resistido para designar o treinamento de força tradicional, conhecido popularmente como musculação, que é um meio de treino que utiliza pesos ou aparelhos em sua execução.

Foram utilizados dois instrumentos na coleta de dados. Para a caracterização da amostra, foi utilizado um questionário sóciodemográfico, visando a identificação do perfil da amostra.

Além disso, foi utilizado o Behavioral Regulation in Exercise Questionnaire Version3 (BREQ-3) de Wilson (2006), traduzido e validado para o português por Guedes e Sofiati (2015). O questionário é composto por 23 afirmações, precedidas pelo enunciado "Por que você pratica exercício físico?", devendo o sujeito anotar o seu nível de concordância através de uma escala Likert de cinco pontos, sendo 0 "nada verdadeiro" e 4 "totalmente verdadeiro". Com os resultados obtidos em cada uma das afirmações, fica possível identificar, ordenar e dimensionar os seis 
estágios do continuum da autodeterminação, tais quais: amotivação - AMOT; motivação extrínseca de regulação externa - REEX; motivação extrínseca de regulação introjetada - REIJ; motivação extrínseca de regulação identificada REID; motivação extrínseca de regulação integrada - REIG; e motivação intrínseca - MOTI. O índice de autodeterminação (IAD) utilizado foi dimensionado através da seguinte fórmula (MOTI + MOTI + MOTI +REIG +REIG + REID) - (REIJ + REEX + REEX + AMOT + AMOT + AMOT) e usou os valores obtidos através dos fatores do BREQ-3. O alfa de Cronbach apresentou coeficientes entre 0,67 e 0,87 (GUEDES E SOFIATI, 2015).

Os instrumentos da pesquisa foram aplicados de forma coletiva pelo pesquisador responsável, antes do treino de cada participante. Primeiro o pesquisador fez o convite, a seguir, entregou os Termos de Consentimentos Livre e Esclarecido (TCLE) para os participantes assinarem e, na sequência, entregou os questionários e explicou como deveria ser respondido, esclarecendo possíveis dúvidas. O pesquisador ficou presente durante todo o tempo da coleta. Esse estudo foi aprovado pelo Comitê de Ética em Pesquisa com o Parecer n 3.401.784.

O banco de dados foi caracterizado por meio da média, desvio padrão (DP) e frequências absoluta e relativa. $O$ teste Kolmogorov-Smirnov foi usado para identificar a normalidade das variáveis contínuas. A fim de comparar as variáveis contínuas entre os sexos, foi usado o teste $t$ para amostras independentes (distribuição normal) ou Mann-Whitney (não paramétrico). O teste Exato de Fisher foi usado para comparar as variáveis categóricas de acordo com os sexos. Para identificar fatores associados aos diferentes domínios do BREQ-3 e ao índice de autodeterminação, foram realizados Modelos Lineares Generalizados, considerando todos os aspectos comportamentais e antropométricos como preditores. Em seguida, os modelos foram ajustados por meio da remoção das variáveis menos explicativas $(>0,05)$ até alcançar o menor valor de AIC (Critério de Informação de Akaike). Todas as análises ocorreram a 5\% de significância estatística, sendo processadas no Statistical Package for the Social Science (SPSS; IBM, Chicago, IL, EUA), versão 25,0.

\section{Resultados e Discussão}

Em média, os participantes apresentaram 33,37 $\pm 10,22$ anos, massa

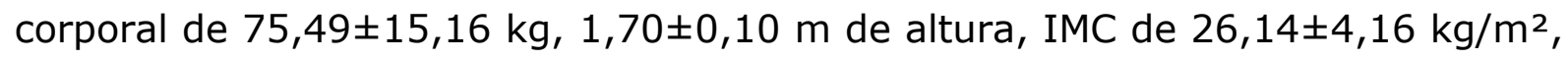

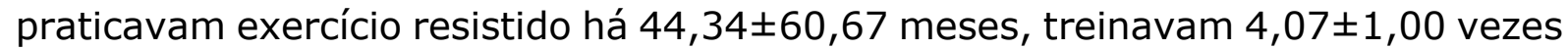
por semana e $72,77 \pm 22,43$ minutos por sessão. Mais da metade da amostra $(56,4 \%)$ era formado por homens e praticantes de outras modalidades esportivas $(53,2 \%)$. As modalidades mais praticadas foram futebol $(18,1 \%)$, corrida $(13,8 \%)$ e caminhada $(8,5 \%)$. Ciclismo $(5,3 \%)$, lutas $(3,1 \%)$ e basquetebol $(2,1 \%)$ também foram mencionados, porém em menores frequências. Sobre os objetivos para a prática de exercícios resistidos, os participantes buscavam saúde $(54,3 \%)$, 
estética (22,3\%), prazer $(11,7 \%)$ e emagrecer $(11,7 \%)$. Em relação aos domínios

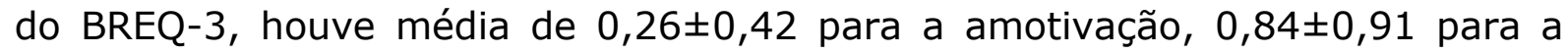

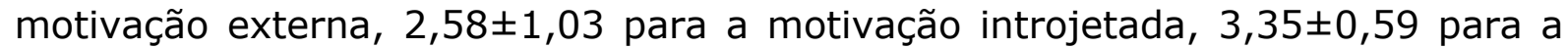
motivação identificada, $3,40 \pm 0,69$ para a integrada e $3,43 \pm 0,65$ para a motivação intrínseca.

A Tabela 1 apresenta a comparação das variáveis do estudo de acordo com o sexo. Ocorreram diferenças estatisticamente significativas entre os sexos, indicando que a média do peso e a altura foram maiores entre os homens, que frequentavam a academia mais vezes na semana, além de serem mais adeptos à prática do futebol. Para as demais variáveis não houve diferenças estatisticamente significativas.

Tabela 1 - Comparação das variáveis do estudo de acordo com o sexo. Dados de praticantes de exercícios resistidos de diferentes academias de ginástica do município de Itapira-SP

\begin{tabular}{|c|c|c|c|c|c|}
\hline \multirow{2}{*}{ Variáveis } & \multicolumn{2}{|c|}{ Masculino $(n=53)$} & \multicolumn{2}{|c|}{ Feminino $(n=41)$} & \multirow{2}{*}{$P$ valor } \\
\hline & Média & DP & Média & DP & \\
\hline Idade & 32,02 & 9,60 & 35,12 & 10,85 & 0,145 \\
\hline Peso (kg) & 81,81 & 14,30 & 67,32 & 12,13 & $<0,001$ \\
\hline Altura (m) & 1,76 & 0,07 & 1,62 & 0,06 & $<0,001$ \\
\hline IMC $\left(\mathrm{kg} / \mathrm{m}^{2}\right)$ & 26,50 & 4,27 & 25,68 & 4,01 & 0,340 \\
\hline Tempo de treino (meses) & 52,34 & 62,92 & 33,99 & 56,71 & 0,147 \\
\hline Frequência (dias/semana) & 4,30 & 0,99 & 3,78 & 0,94 & 0,011 \\
\hline Sessão (minutos) & 73,02 & 19,15 & 72,44 & 26,32 & 0,906 \\
\hline \multicolumn{6}{|l|}{ Domínios da motivação } \\
\hline Amotivação & 0,23 & 0,41 & 0,29 & 0,44 & 0,486 \\
\hline Externa & 0,76 & 0,83 & 0,94 & 1,01 & 0,370 \\
\hline Introjetada & 2,55 & 0,94 & 2,63 & 1,14 & 0,733 \\
\hline Identificada & 3,25 & 0,64 & 3,48 & 0,50 & 0,072 \\
\hline Integrada & 3,40 & 0,64 & 3,41 & 0,76 & 0,958 \\
\hline Intrínseca & 3,43 & 0,65 & 3,41 & 0,65 & 0,887 \\
\hline Índice de autodeterminação & 15,58 & 3,90 & 15,15 & 5,06 & 0,643 \\
\hline Outras modalidades & $\mathbf{n}$ & $\%$ & $\mathbf{N}$ & $\%$ & \\
\hline Futebol & 17 & 32,1 & 0 & 0,0 & $<0,001$ \\
\hline Corrida & 9 & 17,0 & 4 & 9,8 & 0,378 \\
\hline Caminhada & 4 & 7,5 & 4 & 9,8 & 0,725 \\
\hline \multicolumn{6}{|l|}{ Objetivo principal } \\
\hline Saúde & 28 & 52,8 & 23 & 56,1 & 0,836 \\
\hline Estética & 15 & 28,3 & 6 & 14,6 & 0,139 \\
\hline Prazer & 7 & 13,2 & 4 & 9,8 & 0,751 \\
\hline Emagrecer & 3 & 5,7 & 8 & 19,5 & 0,053 \\
\hline
\end{tabular}

$\mathrm{DP}=$ Desvio Padrão; $\mathrm{n}=$ frequência absoluta; $\%=$ frequência relativa.

A Tabela 2 apresenta os fatores associados aos domínios do BREQ-3 e ao índice de autodeterminação. Houve associação estatisticamente significativa nos 
modelos de amotivação, motivação externa, motivação introjetada e motivação identificada. Cada unidade de aumento na frequência de treino reduz 0,091 na média da amotivação, cada aumento unitário no IMC aumenta 0,049 na motivação externa e cada unidade de aumento na sessão de treino adiciona 0,013 na média da motivação introjetada. Observamos também que a média da motivação introjetada foi maior no sexo feminino. Para as demais variáveis dependentes, os preditores presentes nos modelos finais não foram estatisticamente significativos.

Tabela 2 - Modelos finais correspondentes aos preditores dos domínios do BREQ-

3. Dados de praticantes de exercícios resistidos de diferentes academias de ginática do município de Itapira-SP.

\begin{tabular}{|c|c|c|c|c|}
\hline Desfechos & Preditores & $\boldsymbol{\beta}$ & EP & $\mathbf{p}$ \\
\hline \multirow[t]{2}{*}{ Amotivação } & Frequência (dias/semana) & $-0,091$ & 0,044 & 0,041 \\
\hline & Sessão (minutos) & 0,002 & 0,002 & 0,259 \\
\hline \multirow[t]{3}{*}{ Externa } & Frequência (dias/semana) & $-0,131$ & 0,095 & 0,169 \\
\hline & Sessão (minutos) & $-0,006$ & 0,004 & 0,137 \\
\hline & $\operatorname{IMC}\left(\mathrm{kg} / \mathrm{m}^{2}\right)$ & 0,049 & 0,023 & 0,034 \\
\hline \multirow[t]{3}{*}{ Introjetada } & Frequência (dias/semana) & $-0,145$ & 0,106 & 0,176 \\
\hline & Sessão (minutos) & 0,013 & 0,005 & 0,006 \\
\hline & Tempo de treino (meses) & 0,002 & 0,002 & 0,307 \\
\hline \multirow[t]{3}{*}{ Identificada } & Masculino - Feminino & $-0,255$ & 0,123 & 0,040 \\
\hline & $\operatorname{IMC}\left(\mathrm{kg} / \mathrm{m}^{2}\right)$ & 0,014 & 0,015 & 0,369 \\
\hline & Sessão (minutos) & 0,005 & 0,003 & 0,055 \\
\hline \multirow[t]{2}{*}{ Integrada } & Sessão (minutos) & 0,006 & 0,003 & 0,056 \\
\hline & Tempo de treino (meses) & 0,002 & 0,001 & 0,085 \\
\hline \multirow[t]{2}{*}{ Intrínseca } & Sessão (minutos) & 0,005 & 0,003 & 0,106 \\
\hline & Tempo de treino (meses) & 0,002 & 0,001 & 0,061 \\
\hline \multirow[t]{2}{*}{ Autodeterminação } & Frequência (dias/semana) & 0,780 & 0,449 & 0,086 \\
\hline & Tempo de treino (meses) & 0,014 & 0,007 & 0,071 \\
\hline
\end{tabular}

Legenda: $\mathrm{EP}=$ Erro Padrão.

Esse estudo teve como objetivo analisar os níveis motivacionais de praticantes de exercícios resistidos. Observou-se que o maior score ocorreu na 
motivação intrínseca, ou seja, quando o indivíduo está motivado ingressará na atividade por vontade própria, seja por satisfação ou por prazer (RYAN; DECI, 2000). Em pesquisa realizada por Santos et al. (2018), observou-se que os participantes foram motivados intrinsecamente para a prática de atividades físicas. Pessoas que se mantêm em programas de atividade física são motivadas a continuar na prática por mais tempo. O prazer pela prática é uma forte influência para a manutenção da mesma, onde a soma das necessidades torna a atividade física mais significativa para o indivíduo (SANTOS; KNIJNIK, 2006).

No presente estudo houve associação direta entre motivação externa e IMC, sendo maior entre os homens. Isso sinaliza a existência de um estímulo externo para a prática de uma atividade, sendo frequentemente ligados a saúde por conta do sobrepeso, da obesidade ou até mesmo pela busca de uma imagem corporal de um corpo musculoso (MARTINS; DO NASCIMENTO, 2020). Silva Júnior, Abreu e Silva (2017), constataram que os homens apresentam os valores maiores de IMC em comparação às mulheres, o que se associa à maior massa muscular e a um maior percentual de homens com excesso de peso corporal. Por sua vez, a obesidade é considerada pela Organização Mundial da Saúde (OMS) como um problema de saúde que pode levar a complicações psicológicas e físicas (MONTEIRO; NAVARRO, 2010) e está associado ao maior risco de enfermidades crônicas não transmissíveis (GUTTIERRES; MARINS, 2008). A mudança comportamental ou do estilo de vida é uma das formas mais eficazes de tratamento para a obesidade, pois essa intervenção engloba, além de um acompanhamento nutricional e de um suporte psicológico, a prática regular de exercícios físicos (GARVEY et al., 2016).

Outro fator a se considerar a respeito dos homens é que eles se mostraram mais assíduos na prática de exercícios resistidos. É possível que a maior assiduidade por parte dos homens seja por conta da necessidade de se ter um corpo musculoso (MARTINS; DO NASCIMENTO, 2020), atendendo ao modelo característico da cultura corporal ocidental (TATANGELO; RICCIARDELLI, 2013). Essa preocupação com aparência fez com que Dutra et al. (2019) constatassem que praticantes de musculação são, de fato, mais ativos e mais satisfeitos com sua imagem corporal quando comparados com não praticantes.

Um outro achado importante foi que com o aumento da frequência de treino a média da amotivação diminui. É alto o índice de evasão de praticantes de exercícios em academias de ginástica no Brasil (ALBUQUERQUE; ALVES, 2007), sendo crucial os seis primeiros meses de prática, com taxas de evasão chegando em torno de 50\% (SABA, 2001; MARCUS et al., 2000). Gonçalves e Alchieri (2010) acreditam que quanto mais tempo as pessoas conseguem se manter na prática da atividade física menor será a chance de desistirem da prática, o que demonstra uma possível prática na motivação autônoma para a atividade. Santos et al. (2018) identificaram que a amotivação está relacionada ao trabalho, podendo variar de acordo com os objetivos individuais, conforme as necessidades ao longo da vida. 
Além disso, as pessoas apresentam a falta de tempo, a distância entre a academia e suas respectivas casas e a falta de motivação como fatores decisivos para a desistência da atividade física em academias (DE MELLO et al. , 2018). Assim, entre as razões para a amotivação, podemos destacar ainda que as pessoas podem não enxergar resultados, tanto na estética quanto na saúde, uma vez que, para a promoção da saúde, é recomendada a realização de atividades físicas com intensidade de moderada a intensa, na maior parte da semana, de forma contínua e progressiva (PATE et al., 1995).

A motivação introjetada teve associação direta com a duração de cada sessão de treino, salientando que os sujeitos que se apresentaram como intrinsecamente motivados permaneciam mais tempo na academia. Soler et al. (2013) dizem que quanto maior o tempo de prática de atividade física de um indivíduo na academia, mais satisfeito ele está com a sua aparência e melhor é o seu controle sobre o exercício, de modo a sempre se exercitar conforme o programado antes de iniciar a sessão.

Por fim, as mulheres apresentaram maiores níveis de motivação identificada. Em uma revisão de literatura de Mudesto e Da Silva (2018), constataram-se a preocupação com a imagem corporal, pois os estudos encontrados mostraram que a aderência do grupo feminino praticante de exercícios resistidos era a perturbação relacionada à estética, a redução do percentual de gordura e a diminuição do estresse. Portanto, a prática de exercícios resistidos é indicada por auxiliar na manutenção da massa magra e nos ganhos de força, o que aumenta a condição física (GRAVES; FRANKLIN, 2006), melhorando a percepção da imagem corporal nas mulheres.

Os participantes do presente estudo, em sua maioria, eram motivados pela busca da saúde. Pode-se dizer que há uma relação direta entre a prática de atividade física e a saúde (NUNOMOURA; TEIXEIRA; CARUSO, 2004). Santos et al. (2018) confirmaram que os praticantes de atividade física se sentiam motivados devido a fatores também relacionados a saúde. Na revisão de literatura feita por Mudesto e Da Silva (2018), a estética mostrou-se como fator relevante, seguido pela saúde, estresse e bem-estar.

Apesar destes achados contribuírem para uma melhor compreensão dos níveis motivacionais em praticantes de exercícios resistidos, o estudo apresenta algumas limitações. A primeira delas é que os resultados não são generalizáveis para além da amostra estudada, pois não se trata de uma amostra representativa. Em segundo, a pesquisa não levou em consideração a classe social dos participantes. Nesse sentido sugere-se que os próximos estudos levem em consideração outras variáveis em que façam uma análise longitudinal do comportamento dos níveis motivacionais em praticantes de exercícios resistidos. 


\section{CONSIDERAÇÕES FINAIS}

As academias de ginástica tem sido a escolha recorrente para a prática de exercícios físicos de um número elevado de pessoas. Este estudo mostrou que os praticantes de exercício resistido buscam, em sua maioria, saúde e estão motivados intrinsecamente para a prática.

É importante considerar a motivação pessoal que leva cada indivíduo a frequentar esse ambiente, de forma a entender o nível de motivação desse aluno no momento que ele ingressa na atividade, adotando estratégias eficazes para a manutenção dessas pessoas dentro das academias de ginástica.

Essa pesquisa pode trazer informações relevantes aos proprietários de academias que poderão utilizá-las para melhorar o serviço ofertado à população que procura por esses espaços para a prática de exercícios resistidos.

\section{NotAS}

\section{CONFLITOS DE INTERESSE}

Os autores não têm conflitos de interesse, incluindo interesses financeiros específicos e relacionamentos e afiliações relevantes ao tema ou materiais discutidos no manuscrito.

\section{Autoria e COAUTORIA}

Os autores declaram que participaram de forma significativa na construção e formação desde estudo, tendo, enquanto autor, responsabilidade pública pelo conteúdo deste, pois, contribuíram diretamente para o conteúdo intelectual deste trabalho e satisfazem as exigências de autoria.

Elisabeth Cristina Canola - Coleta e tratamento dos dados (responsável pelos experimentos, pacientes, organização dos dados); Análise / interpretação (responsável pela análise estatística, avaliação e apresentação dos resultados); Levantamento da literatura (participou da pesquisa bibliográfica e levantamento de artigos); Redação (responsável por escrever uma parte substantiva do manuscrito).

João Guilherme Cren Chiminazzo - Concepção e desenvolvimento (desde a ideia para a investigação ou artigo, criou a hipótese); Desenho metodológico (planejamento dos métodos para gerar os resultados); Supervisão (responsável pela organização e execução do projeto e da escrita do manuscrito); Análise / interpretação (responsável pela análise estatística, avaliação e apresentação dos resultados); Redação (responsável por escrever uma parte substantiva do manuscrito); Revisão crítica (responsável pela revisão do conteúdo intelectual do manuscrito antes da apresentação final). 


\section{REFERÊNCIAS}

ALBUQUERQUE, Cristina Lucia F., Alves, Ruziano S. A evasão dos alunos das academias: Um estudo de caso no centro integrado de estética e atividade física - CIEAF, na cidade de Caicó - RN. Dominium Revista Científica da Faculdade de Natal, Natal, v. 1, n. 5, p. 133, jan./abr. 2007.

BAVOSO, Daniel; GALEOTI, Lívia; MONTIEL, José Maria; CECATO, Juliana Francisca. Motivação e autoestima relacionada à prática de atividade física em adultos e idosos. Revista Brasileira de Psicologia do Esporte, Brasília, v. 7, n. 2, out. 2017.

CARNEIRO, Luís; GOMES, A. Rui. Querer fazer exercício e fazer exercício: papel dos fatores pessoais e psicológicos. Revista Iberoamericana de Psicologia del Ejercicio y el deporte, v. 11, n. 2, p. 253-261, 2016.

DECI, Edward L.; RYAN, Richard M. Autonomy and need satisfaction in close relationships: Relationships motivation theory. In: WESTEIN, N. (Ed.) Human motivation and interpersonal relationships, Dordrecht: Springer, p. 53-73, 2014.

DECI, E. L.; RYAN, R. M. Intrinsic Motivation and Self-Determination in Human Be-havior. New York: Plenum Press, 1985.

DUTRA, Sebastião R. R.; DA SILVA, D. C.; CORREA, A. A. M.; LAVORATO, V. N. Nível de satisfação com imagem corporal em homens e mulheres praticantes e não praticantes de musculação. Caderno Científico UNIFAGOC de Graduação e Pós-Graduação, v. 5, n. 1, 2021.

FERNANDES; Helder Miguel; RAPOSO, José Vasconcelos. Continuum de autodeterminação: validade para a sua aplicação no contexto desportivo. Estudos de Psicologia, v. 10, n. 3, p. 385-395, 2005.

FERREIRA, Ana Clara Prado; De SOUZA, Renato Ferreira. Revisão sistemática de literatura sobre a questão motivacional no esporte. Revista FSA, v. 16. N. 2, 2019.

GARVEY, W. Timothy; MECHANICK, Jeffrey I.; BRETT, Elise M.; GARBER, Alan J.; HURLEY, Daniel L.; JASTREBOFF, Ania M.; NADOLSKY, Karl; POLLACK, Rachel Pessah; PLODWOSKI, Raymond. American Association of Clinical Endocrinologists and American College of Endocrinology comprehensive clinical practice guidelines for medical care of patients with obesity. Endocrine Practice, v. 22, n. 3, p. 1-203, 2016.

GONÇALVES, Marina Pereira; ALCHIERI, João Carlos. Motivação à prática de atividades físicas: um estudo com praticantes não-atletas. Psico-USF. v. 15, n. 1, p. 125-134, 2010.

GRAVES, James E.; FRANKLIN, Barry. A. Treinamento Resistido na saúde e reabilitação, 2. ed. Rio de Janeiro: Revinter, 2006.

GUEDES, Dartagnan Pinto; SOFIATI, Sandro Lucas. Tradução e validação psicométrica do Behavioral Regulation in Exercise Questionare para uso em adultos brasileiros. Revista Brasileira de Atividade Física \& Saúde, v. 20, n. 4, 2015.

GUTTIERRES, Ana Paula Muniz; MARINS, João Carlos Bouzas. Os efeitos do treinamento de força sobre os fatores de risco da síndrome metabólica. Revista Brasileira Epidemiologia, Juiz de Fora, v. 11, n. 1, p. 147-158, 2008. 
KOPP, P. M.; SENNER, V.; KEHR, H. M.; GRÖPEL, P. Achievement motive, autonomous motivation, and attendance at fitness center: A longitudinal prospective study. Psychology of Sport and Exercise, 51, 101758, 2020.

MARCUS, Bess H.; DUBBERT, Patricia M.; FORSYTH, LeighAnn H.; MCKENZIE, Thomas L.; STONE, Elaine J.; DUNN, Andrea L.; BLAIR, Steven N. Physical activity behavior change: issues in adoption and maintenance. Health Psychology, v. 19, n. 1, p. 32, 2000.

MARTINS, Alberto Mesaque; DO NASCIMENTO, Adriano Roberto Afonso. Imagem corporal masculina: revisão integrativa da produção científica latino-americana (2005-2019). Motrivivência, v. 32, n. 63, p. 01-23, 2020.

MATSUDO, Sandra Mahecha; MATSUDO, Victor Keihan Rodrigues; NETO, Turíbio Leite Barros. Efeitos benéficos da atividade física na aptidão física e mental durante o processo de envelhecimento. Revista Brasileira de Atividade Física \& Saúde, v. 5, n. 2. p. 60-76, 2000.

MONTEIRO, Eduardo Chaves; NAVARRO, Francisco. Leptina, obesidade e exercício físico. Revista Brasileira de Obesidade, Nutrição e Emagrecimento, São Paulo, v. 4, n. 19, p. 54-60, 2010.

MOUTÃO, João; ALVES, Susana; CID, Luís. Contributo da teoria da autodeterminação na predição da vitalidade e adesão ao exercício físico. Revista Gymnasium, v. 1, n. 4, p. 14, 2012.

MUDESTO, Moísa Anselmo; DA SILVA, Giuliano Roberto. Fatores que levam a adesão do grupo feminino na prática de musculação: uma revisão de literatura. Revista Gestão Universitária, v. 9, 2018.

NAHAS, M. V. Atividade física, saúde e qualidade de vida: conceitos e sugestões para um estilo de vida ativo. 3 ed. Londrina: Midiograf, 2003.

NUNOMOURA, Myrian; TEIXEIRA, Luis Antonio Cespedes; CARUSO, Mara Regina Fernades. Nível de estresse em adultos após 12 meses de prática regular de atividade física. Revista Mackenzie de Educação Física e Esporte, v. 3, n. 3. P. 125-134, 2004.

PATE, Russel R.; MACERA, Carol; BOUCHARD, Claude; HEALTH, Gregory W. Physical activity and public health: a recommendation from the Centers for Disease Control and Prevention and the American College of Sports Medicine. The jornal of the American Association, v. 273, n.5, p. 402-07, 1995.

RUFINI, Sueli Édi.; BZUNECK, José Aloyseo; OLIVEIRA, Katya Luciane. A qualidade da motivação em estudantes do ensino fundamental. Paidéia, v. 22, n. 51, p. 53-62, 2012.

RYAN, Richard M.; DECI, Edward L. Intrinsican dextrinsic motivations: classic definitions and new directions. Contemporary Education Psychology, New York, v. 25, n.1, p. 54-67, 2000.

RYAN, Richard M.; DECI, Edward L. Self-determinations theory: Basic psychological needs in motivation, development, and welness. New York: Guilford Publications, 2017.

SABA, Fábio. Aderência: A prática do exercício físico em academias. São Paulo: Manole, 2001.

SAMULSKI, D. M.; COSTA, I. T.; AMPARO, L. P. et al. Atividade física, saúde e qualidade de vida. In: SAMULSKI, D. M. (Org.). Psicologia do Esporte: conceitos e novas perspectivas. 2. ed. Barueri: Manole, 2009. 
SANTOS, Francielle Carvalho; OLIVEIRA, Renata Aparecida Rodrigues; CORRÊIA, Alexandre Augusto Macêdo; FERREIRA, Elizângela Fernades. Motivação para a prática de atividades físicas: um estudo com praticantes de musculação. Revista Científica Fagoc Multidisciplinar, v. 3, 2018.

SANTOS, Susan Cotrim; KNIJNIK, Jorge Dorfman. Motivos à prática de atividade física na vida adulta intermediária I. Revista Mackenzie de Educação Física e Esportes, v. 5, n.1, p. 23-34, 2006.

SCHULTZ, Duane; SCHULTZ, Sydney Ellen. Teorias da personalidade. São Paulo: Pioneira Thomson, 2002.

SILVA JÚNIOR, Ronaldo; ABREU, Wilson César; SILVA, Richard Fernado. Composição corporal, consumo alimentar e hidratação de praticantes de musculação. Revista Brasileira de Nutrição Esportiva, São Paulo, v. 11, n. 68, p. 936-946, 2017.

SILVA, Iranzé Oliveira; PEREIRA, Lúcia Coelho Garcia; VENÂNCIO, Patrícia Espíndola Mota. Motivação à prática de musculação em adultos jovens de 18 a 25 anos. Coleção Pesquisa em Educação Física, Várzea Paulista, v. 15, n. 1, p. 119-126, 2016.

SILVA, Letícia Campos; CAMARGOS, Gustavo Leite; CORREA, Alexandre; DA SILVA, Davi Correia. Influência de fatores motivacionais na prática da musculação. Caderno Científico UNIFAGOC de Graduação e Pós-Graduação, v. 5, n. 1, 2021.

SILVA, Rodrigo Sinnott; DA SILVA, Ricardo Azevedo; SOUZA, Luciano; TOMASI, Elaine. Atividade física e qualidade de vida. Ciência \& Saúde Coletiva. v. 15, n. 1, p. 115-120, 2010.

SOLER, Patrícia Tatiana; FERNANDES, Helder Miguel; DAMASCENO, Vinícius Oliveira; NOVAES, Jefferson Silva. Vigorexia e níveis de dependência de exercício em frequentadores de academias e fisiculturistas. Revista Brasileira de Medicina do Esporte. São Paulo, v. 19, n. 5, 2013.

TATANGELO, Gemma L.; RICCIARDELLI, Lina A. A qualitative study of preadolescent boys' and girls' body image: Gendered ideals and sociocultural influences. Body image, v. 10, n. 4, p. 591-598, 2013.

WILLIAMS, David. M., DUNSIGER, Shira, CICCOLO, Joseph T., LEWIS, Beth A., ALBRECHT, Anna E., MARCUS, Bess. H. (2008). Acute affective response to a moderateintensity exercise stimulus predicts physical activity participation 6 and 12 months later. Psychology of sport and exercise, v. 9, n. 3, p. 231-245, 2008. 
Artigo submetido ao sistema de similaridade Turnitin ${ }^{\circledR}$.

A revista Conexões utiliza a Licença Internacional Creative Commons Atribuição 4.0, preservando assim, a integridade dos artigos em ambiente de acesso aberto.

A Revista Conexões é integrante do Portal de Periódicos Eletrônicos da Unicamp e associado/membro das seguintes instituições:

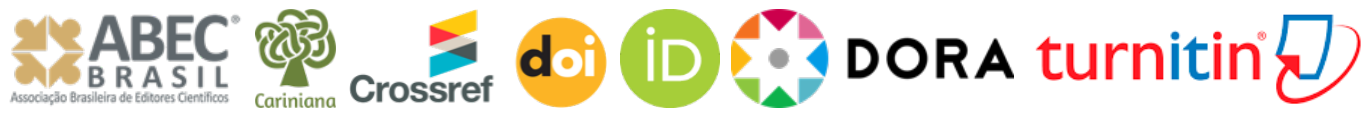

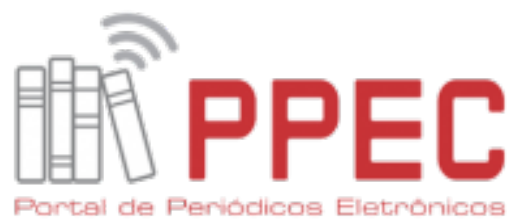

Cientificos de UNICAMP 